\title{
Emprendimiento e instituciones: caso de estudio de la población víctima en posconflicto ${ }^{1}$
}

\section{Entrepreneurship and institutions: case study of the victim population in post-conflict}

CÉrbulo Andrés Cifuentes García ${ }^{2}$

AlexandRa Hoyos BRavo ${ }^{4}$

\section{Resumen}

En Colombia, después de más de cincuenta años de conflicto armado interno, se produjo la firma del acuerdo de paz con las FARC. En este nuevo contexto, el emprendimiento se presenta como una posible herramienta para la generación de ingresos y el empoderamiento económico de la población víctima del conflicto. El presente artículo tiene como objetivo conocer el efecto de las acciones realizadas por las instituciones (actores del ecosistema) en la actividad emprendedora de la población víctima en posconflicto, para ello se realiza un análisis descriptivo y de algunas variables como la existencia de infraestructura productiva, entre otras. La estimación del modelo mostró que las variables de programas de apoyo financiero, el acceso a capacitación, las acciones para formalizar 0 normalizar los emprendimientos y el acceso a préstamos de la banca comercial, no son estadísticamente significativas para explicar la actividad emprendedora de esta población.

Palabras clave: emprendimiento, instituciones, víctimas, posconflicto.

1. Este artículo de investigación es resultado del proyecto "Emprendimiento y posconflicto: una mirada a Risaralda y el Valle del Cauca, fase 3: Rutas alternas de emprendimiento para la población víctima del conflicto".

2. Economista, Maestría en Administración de Empresas. Docente Facultad de Administración, Contabilidad y Finanzas de la Fundación Universitaria Católica Lumen Gentium - UNICATÓLICA. Grupo de Investigación Interdisciplinario en Gestión Organizacional - GIICO. Correo electrónico: ccifuentes@unicatolica.edu.co. Orcid: https:// orcid.org/ 0000-0003-2826-6284

3. Economista, Maestría en Economía. Docente Facultad de Administración, Contabilidad y Finanzas de la Fundación Universitaria Católica Lumen Gentium - UNICATÓLICA. Grupo de Investigación Interdisciplinario en Gestión Organizacional - GIICO. Correo electrónico: ahoyosb@unicatolica.edu.co. Orcid: https:// orcid.org/0000-0003-1330-6546

4. Administradora de Empresas, Maestría en Administración de Empresas. Docente Facultad de Administración, Contabilidad y Finanzas de la Fundación Universitaria Católica Lumen Gentium - UNICATOLICA. Grupo de Investigación Interdisciplinario en Gestión Organizacional - GIICO. Correo electrónico: sgonzalez@unicatolica.edu.co. Orcid: http:// orcid.org/ 0000-0001-7205-3919 


\section{Abstract}

After more than fifty years of internal armed conflict, the signing of the peace agreement between FARC guerrillas and the Colombian government. In this context, the entrepreneurship rises as a possible tool for income generation and economic empowerment for the victims of conflict hit by violence. The article assesses the effects of the institutions' actions (ecosystem actors) on the entrepreneurial activity of the postconflict victim population, through a descriptive and empirical analysis of some selected variables with cross-sectional data. The econometric analysis suggests that financial support programs, access to training, actions to formalize or normalizing entrepreneurship, and access to commercial bank loans are not statistically significant to explain the entrepreneurial activity of this population.

Keywords: entrepreneurship, institutions, victims, post-conflict.

\section{Introducción}

En el emprendimiento de un país intervienen diferentes elementos del contexto (Desai et al., 2013). En Colombia el emprendimiento se ha desarrollado bajo un escenario de necesidad, falta de oportunidades - atenuadas por el conflicto armado- y, por ende, con bajos niveles de capital humano. Este conjunto de elementos plantea para la región una serie de retos con el fin de promover, fomentar y apoyar el emprendimiento, entendiendo que este genera un efecto positivo en la economía y en la sociedad en general (Coyne et al.,
2010; Zietarski, 2018). Colombia ha vivido por más de 50 años el conflicto interno; en septiembre de 2016 se firma un cese al fuego con las Fuerzas Armadas Revolucionarias de Colombia (FARC) y tras ese cese de hostilidades surgen programas por parte del gobierno con el fin de compensar a las víctimas en este contexto de posconflicto y contribuir a la superación de los hechos vividos.

Con base en lo anterior, se identifica que el éxito empresarial tiene una relación con el contexto institucional (Fuentelsaz y González, 2015); en este sentido, Acs (2006) indica que la decisión de emprender está definida por condiciones empresariales en el país, entendiendo estas como factores que afectan el entorno empresarial, tales como el financiamiento, los aspectos sociales y culturales; además de la existencia de las condiciones generales como la infraestructura y las políticas, entre otros.

Lo anterior fue ratificado por CortésSánchez (2018), quien al analizar el desarrollo empresarial en algunas empresas colombianas, llegó a la conclusión de que hay una relación significativa entre la calidad de las instituciones ${ }^{5}$ y el emprendimiento productivo. Teniendo en cuenta esto, aunque las capacidades propias del emprendedor inciden en el desarrollo empresarial, es importante conocer que en un contexto social y económico en el que hay pocas oportunidades de trabajo, altos niveles de pobreza y desigualdad y existe un conflicto interno, es común que el emprendimiento desarrollado presente bajos

5. Según Kantis, Federico y Magendzo (2015) la institucionalidad está representada en los diferentes actores que hacen parte y actúan en los ecosistemas de emprendimiento. En Colombia, estos ecosistemas tienen presencia regional y local; además, estos actores se enfocan en la generación de política pública, formación de talento humano, financiamiento de los emprendimientos, apoyo técnico y facilitan la consolidación de redes empresariales. 
niveles de crecimiento, ausencia de estrategia, bajas habilidades y competencias, que finalmente no contribuyen al crecimiento ni a la prosperidad de la unidad productiva.

En este tema, Desai et al. (2013) concluyen que, aunque las capacidades internas y la elección de la actividad influyen en un emprendimiento productivo, es necesario contar con instituciones sólidas y comprometidas con el desarrollo empresarial, dispuestas a asumir el reto de fortalecerse y responder ante las necesidades de un entorno cambiante. En esto concuerdan Vorley y Williams (2017), y además mencionan que es importante superar los rezagos económicos, sociales, políticos y culturales para promover un tipo de emprendimiento productivo; en este sentido, Zietarski (2018), Coyne et al. (2010) y Sobel (2008) analizan que las instituciones tienen la capacidad de influir para que se desarrolle un emprendimiento productivo, improductivo o destructivo. En la medida que los emprendedores perciban respaldo, facilidad para ejercer su actividad y una infraestructura adecuada, optarán por desarrollar emprendimiento de impacto en la región, pero si, por el contrario, hay barreras impositivas, administrativas, no hay regulaciones para el goce de sus derechos, se desarrollará con mayor facilidad un tipo de emprendimiento improductivo y, en algunos casos, destructivo.

Teniendo en cuenta el contexto de posconflicto y el emprendimiento del país, es necesario enfocar el análisis desde las instituciones y su papel en el desarrollo emprendedor de los colombianos, por lo cual el objetivo de este texto será conocer el efecto de las acciones realizadas por las instituciones en la actividad emprendedora de la población víctima. Para el desarrollo de esta investigación se utilizaron datos de corte transversal recolectados a 200 personas víctimas del conflicto armado, ubicados en Buga y Tuluá (Valle del Cauca - Colombia); se utiliza una matriz de correlación para valorar la relación entre las variables y se plantea un modelo de probabilidad lineal para el análisis empírico. Se plantea la hipótesis de que las acciones institucionales afectan de manera significativa la actividad emprendedora en la población sujeto de estudio.

El documento se desarrolla inicialmente con los antecedentes - marco de referencia, luego se presenta el marco teórico o conceptual y la metodología; posteriormente se exponen las conclusiones y recomendaciones y finalmente se registran las referencias bibliográficas usadas.

\section{Antecedentes - marco de referencia}

La revisión de la literatura se centra en los documentos que analizan la eficiencia del emprendimiento como herramienta del desarrollo económico y los efectos e influencia de las instituciones en la dinámica emprendedora, en un contexto de posconflicto.

Algunos autores plantean que a pesar de que el emprendimiento ha sido considerado como motor de desarrollo, también es claro que puede presentar efectos negativos en la economía. En este sentido, Coyne et al. (2010) buscan explicar que la labor emprendedora puede conducir al estancamiento económico. Para demostrar este punto, recurren al análisis de los procesos que llevan a cabo los emprendedores improductivos con ejemplos puntuales en países desarrollados y en vía de desarrollo, los cuales terminan generando estancamiento económico en un inicio y finalmente un decrecimiento económico en el largo plazo. Consideran que el emprendimiento productivo trae consigo actos que suman al arbitraje en los negocios y a la innovación 
que genera un aumento de la eficiencia y del crecimiento económico del país. Afirman también que el emprendimiento productivo es positivo y promueve el crecimiento económico mientras el emprendimiento no productivo es negativo y conduce al estancamiento 0 declive económico. Finalmente indican que la permanencia del emprendimiento productivo crea un mayor espíritu empresarial productivo, pero se da una situación inversa cuando es dominante el emprendimiento improductivo. En pocas palabras, las oportunidades tanto productivas como improductivas están ligadas a la acción previa de los emprendedores, siendo la pregunta de fondo: ¿de dónde provienen estas actividades?

Por otra parte, mencionan que la capacidad de cualquier país para lograr y sostener el desarrollo depende de si los beneficios generados por las actividades empresariales productivas superan los efectos negativos de la actividad empresarial no productiva. Esta relación explica las diferencias entre países y también ayuda a explicar por qué algunos países están abrumados por el subdesarrollo económico. En estas circunstancias, los emprendedores canalizan su talento de acuerdo con las reglas del juego, dependiendo de si las instituciones fomentan implícita o indirectamente el emprendimiento improductivo/ destructivo o productivo.

En este orden de ideas, las instituciones podrían incentivar el emprendimiento productivo y hacer que el emprendimiento improductivo sea más difícil de llevar a cabo y menos atractivo. Si bien es posible tener emprendimientos tanto productivos como no productivos, lo importante es si los empresarios productivos son dominantes o no. Si los empresarios destructivos dominan el área de negocios, hay una tendencia a los efectos negativos que pueden conducir al declive económico y al estancamiento. La falta de crecimiento económico puede atribuirse no solo a la carencia de insumos empresariales, sino también a los incentivos institucionales ofrecidos a los empresarios improductivos/ destructivos.

En cuanto a la importancia de las instituciones en la dinámica del emprendimiento, Aidis et al. (2008) abordan la relación de las instituciones y el desarrollo empresarial en Rusia, teniendo en cuenta los aportes de Baumol (1993) y North (1990) sobre el apoyo y promoción de las instituciones como efecto positivo para el desarrollo empresarial. El estudio consiste en comparar el desarrollo y espíritu empresarial con países participantes del Global Entrepreneurship Monitor (GEM) centrándose en Polonia y Brasil. Con datos de las bases de GEM de Rusia, Polonia y Brasil, realizaron regresiones para evaluar diferentes variables relacionadas con el desarrollo económico, actividad emprendedora y las características demográficas asociadas a los participantes de la muestra.

Los autores encontraron que las tasas de entrada empresarial, en países que habían salido de una estructura socialista, son bajas y particularmente la tasa en Rusia. De igual forma, los resultados muestran que existen condiciones que generan mayores barreras de entrada en este país, en comparación con Polonia y Brasil. Así mismo, se reduce la probabilidad de emprender en personas sin experiencia emprendedora o empresarial, además que es más frecuente que los emprendedores puedan desistir tras algún fracaso o problema en la puesta en marcha de la empresa, mayormente en Rusia.

De acuerdo con el estudio, ante la debilidad de las instituciones los empresarios necesitan apoyarse en las redes empresariales, lo 
cual hace que los hombres, las personas con mayor educación y edad tengan mayores posibilidades de emprender, por las redes de trabajo que han consolidado. Y aunque las redes son importantes, son insuficientes ante los requerimientos institucionales de los procesos de desarrollo empresarial (Aidis et al., 2008).

De la misma forma, Fuentelsaz y González (2015) llevaron a cabo un estudio sobre el fracaso empresarial basándose en dos factores, principalmente: el contexto institucional y la calidad del emprendimiento; para ello utilizan una muestra de 69 países durante el periodo 2007-2012. Dentro de sus principales conclusiones, los autores indican que el contexto institucional incide de forma importante en el éxito del emprendimiento: si el entorno institucional es favorable, con unas reglas de juego claras y bien definidas, el emprendedor llevará a cabo su actividad con seguridad. Por ende, "la supervivencia de las empresas no solo dependerá de las características de las mismas o del producto que se lanza al mercado, sino que el entorno es también un elemento fundamental" (p. 79). Al igual que otros autores, indican que las

políticas implantadas en los últimos años tendientes a promover el emprendimiento de cualquier tipo, con independencia de sus características y probabilidad de éxito futuro, deberían revisarse, centrando los recursos disponibles en aquellas propuestas con mayores opciones de salir adelante. (p. 79)

En este mismo tema, en el marco de países que atraviesan procesos de transición y postguerra, Dzafic y Babajic (2016) realizaron un estudio sobre la importancia de esta institucionalidad para mejorar la dinámica de los empresarios y emprendedores en Bosnia y Herzegovina, utilizando datos de 250 empresas y emprendimientos pequeños y medianos. En ese sentido, los autores aseguran que el éxito de una empresa también depende del apoyo que le brinde el Estado, que mediante sus regulaciones puede generar un efecto positivo al crear un clima atractivo y más fácil para las empresas; aunque puede generar un efecto negativo, al obstaculizar el establecimiento y desarrollo de estas; así, la intervención del Estado en la promoción de la empresarialidad también podría conllevar a la formación de emprendimientos improductivos o destructivos; de igual manera, los resultados empíricos confirmaron que los encuestados no estaban satisfechos con el papel del Estado en la eliminación de obstáculos regulatorios y administrativos para los negocios, aunque el sector público consideraba apropiado el rol del Estado en el desarrollo del emprendimiento, mientras que el sector privado considera que las herramientas gubernamentales son malas e insuficientes.

Este hallazgo va en concordancia con lo encontrado por LêKhang y Thành (2018), al concluir que el gasto público tiene una relación positiva con el espíritu empresarial; mientras que la gobernanza tiene un efecto negativo en las iniciativas emprendedoras formales, que puede explicarse por la presencia de los vacíos legales en las políticas públicas, que generan un mayor incentivo a la creación de autoempleo informal; esto deja claro que las instituciones tienen efectos importantes en el emprendimiento de sus regiones. En el mismo contexto, Vorley y Williams (2017) realizaron una investigación en Kosovo, el cual tiene el reto de generar instituciones que le permitan lograr un desarrollo económico y social, fomentando un espíritu empresarial que contribuya a la generación de procesos de emprendimiento y competitividad. Los autores buscan "examinar el papel del espíritu empresarial y la competitividad como aspectos integrales para hacer más resilientes las localidades" (p. 2). 
La investigación empírica fue desarrollada con revisiones de documentos y entrevistas semiestructuradas. Los hallazgos ratifican la relación de las instituciones para promover u obstaculizar el espíritu emprendedor y el mismo emprendimiento en sí; adicionalmente, el contexto de transición de un modelo de gobierno y económico a otro, deja un sinnúmero de retos y rezagos en el campo empresarial, económico e institucional; esto es corroborado por Joseph et al. (2019), que en su investigación sobre el crecimiento de los negocios en Irak afirma que las condiciones comerciales y sociales externas, afectadas generalmente en un contexto de conflicto, como la seguridad y el comercio internacional, son elementos facilitadores u obstaculizadores para el emprendimiento. Por lo que es frecuente encontrar sectores económicos pequeños y poco eficientes, altos niveles de dependencia de importaciones, altas regulaciones o barreras para el desarrollo empresarial y un arraigo cultural hacia la dependencia del Estado por parte de los ciudadanos.

Por último, Vorley y Williams (2017) manifiestan que este tipo de elementos deben superarse a partir de estrategias y políticas institucionales en el país, de tal forma que existan las condiciones para fomentar un espíritu emprendedor productivo que sea competitivo para la región.

Para Colombia, Cortés-Sánchez (2018) realizó un análisis exploratorio sobre la fortaleza institucional, la construcción de paz y el emprendimiento del país, utilizando datos disponibles de 23 de los 32 departamentos para elaborar tres índices compuestos basados en evaluaciones internacionales: Índice de Fortaleza Institucional: Índice de Construcción de la Paz (basado en el Índice de Paz Negativo y el Índice de Paz Positivo) y el Índice de Emprendimiento Productivo.
El autor concluye que, en el país, las instituciones han sido históricamente extractivas, dando origen a un emprendimiento destructivo y al conflicto armado. Además, demostró que existe una correlación significativa entre fortaleza institucional y emprendimiento productivo, y entre población y emprendimiento productivo; por ende, la existencia de instituciones sólidas e inclusivas fomenta la creación de empleos formales, derechos de propiedad y eficiencia del mercado. Esto se observó de manera consistente en los departamentos de la región andina, donde se encuentran la mayoría de las ciudades principales.

\section{Marco teórico o conceptual}

\section{Emprendimiento}

El emprendimiento es una herramienta importante para la dinámica económica. De acuerdo con Shumpeter (1911, como se citó en Sanders y Weitzel, 2013), el emprendedor es un factor indispensable para la innovación y la dinámica capitalista, creando nuevas variaciones (productos, procesos, modelos de negocio) en la economía; estas nuevas empresas innovadoras compiten con empresas establecidas, lo que obliga a estas últimas a mejorar o modificar su producción, que pueden llegar a la liquidación si esto no se hace con éxito (Schumpeter, 1934, 1942, como se citó en Stam, 2009).

Kirzner (1973, como se citó en Sanders y Weitzel, 2013) señala el rol que tiene la meta de ganancias del emprendedor para mantener el equilibrio del mercado. Dado que se supone que el emprendimiento promueve más oportunidades, cualquier cambio de un emprendedor altera el entorno económico y brinda oportunidades para ajustes adicionales para otros emprendedores; además, es probable que la actividad empresarial cree riqueza e im- 
pulse el crecimiento del mercado y la creación de nichos que antes no existían, proporcionando oportunidades de ingreso y expansión de este mercado a nuevos emprendedores (Holcombe, como se citó en Stam, 2009).

Dado lo anterior, el emprendimiento está ligado al crecimiento del mercado y por ende se supone que impacta directamente en el crecimiento económico y en el mejoramiento del nivel de vida de los habitantes de un país; sin embargo, en Latinoamérica, el emprendimiento presenta diferentes retos y características que son correspondientes a la dinámica económica y social de la región; la mayoría de estos emprendimientos cuenta con bajos niveles de innovación, bajo potencial de crecimiento y poca generación de empleo. En términos de capital humano, no tienen las habilidades para generar mayores beneficios de los que podrían obtener trabajando como empleados asalariados (Sanguinetti et al., 2013). El emprendimiento de los países de esta región no presenta altos niveles de innovación; si se compara la productividad con otros países más desarrollados en cuanto a nuevos productos o servicios, la implementación de Investigación y Desarrollo (I+D) y la generación de patentes, es notable la brecha en tecnología, innovación y productividad (García, 2016).

\section{Emprendimiento, conflicto y posconflicto}

Los efectos macroeconómicos de los conflictos fueron planteados por Brainard y Chollet (2007), quienes indicaron que entre las consecuencias más importantes asociadas al nivel de pobreza de un país está un mayor riesgo de conflicto. Los países pobres tienen muchas más probabilidades que los países ricos de sufrir una guerra civil. Los autores sugieren que para un país en el percentil 50 de ingresos (como Irán), el riesgo de un conflicto civil en cinco años es del 7 al $11 \%$; mientras que en los países del percentil 10 (como Ghana o Uganda), el riesgo aumenta entre el 15 y el $18 \%$. Los efectos se pueden observar en términos de la desviación de recursos económicos hacia la seguridad, los altos costos provocados por la perturbación de los mercados, y el aumento del riesgo y la incertidumbre (Brück et al., 2013a).

El estudio del emprendimiento en el conflicto y en el posconflicto es escaso. Dada la importancia particular de los conflictos violentos en los países en desarrollo, el concepto de emprendedor, según Brück et al. (2013b), debe ser más amplio ${ }^{6}$, diferente al definido en los países desarrollados (donde el modelo del emprendedor en ciernes que trabaja se basa en un individuo que inicia su labor en un garaje para terminar consolidando una empresa multinacional). Naudé (2009) señala que el conflicto armado tiene un efecto devastador en el entorno empresarial; además, la actividad empresarial es a menudo ardua durante el conflicto, pero tiene que ajustarse de formas que no son necesariamente eficientes para la economía en desarrollo; así mismo, los empresarios pueden beneficiarse del conflicto, pero también pueden contribuir a la paz y a la reconstrucción en el posconflicto.

Efectos del emprendimiento en la economía: Institucional ismo y emprendimiento productivo, improductivo y destructivo

Sauka (2008) indica que el emprendimiento puede entenderse de varias formas y que no 
todo lo que se etiqueta como "empresarial" es realmente deseable. Además señala que Baumol (1993) definía a los emprendedores simplemente como personas innovadoras que generaban ganancias o aumentaban su poder y prestigio, y que no se podía esperar que la cantidad de actividades que tuvieran que realizar para ello contribuyeran a la economía. Para Sauka (2008) la pregunta clave sería la creación de valor por parte de los emprendedores, indicando la necesidad de diferenciar las actividades "positivas" o "negativas"; en palabras de Baumol (1990, 1993), el emprendimiento productivo, improductivo y destructivo.

Almodóvar (2018) plantea que el emprendimiento se ha relacionado extensamente con los beneficios socioeconómicos que conlleva, en términos positivos; sin embargo, indica que algunos autores como Audrestsch (2009) y Almodóvar (2016) mencionan que "parte de los investigadores y de los poderes públicos han relegado a un segundo plano el impacto real del emprendimiento, centrándose principalmente en su promoción" ( $p$. 226), generalizando, sin tener en cuenta sus características específicas según la actividad económica, las condiciones del emprendedor, entre otros factores. Así mismo, Almodóvar (2018) indica que existen dudas sobre el desempeño económico del emprendimiento genérico, de acuerdo con Sautet (2013), Liñán y Fernández-Serrano (2014) y Vivarelli (2016).

Aunque algunos autores como Urbano y Aparicio (2016) plantean que el emprendimiento sí es una herramienta eficaz. Esta divergencia en los resultados puede deberse a los diferentes indicadores o índices utilizados para medir los impactos (Stenholm et al., 2013, como se citó en Almodóvar, 2018). Adicionalmente, Almodóvar define que "es posible que esta disparidad de planteamientos refleja que, bajo ciertas circunstancias, el emprendimiento genérico pueda ser aplicable y que derive en beneficios económicos, a pesar de que, si se aplicara un emprendimiento específico, el beneficio sería todavía mayor" (p. 241). En este sentido, según el autor, "la política pública puede elegir si fomentar el emprendimiento genérico de impacto positivo, o el específico de impacto positivo aún mayor" (p. 241), todo dependerá del tipo de economía y de las condiciones existentes para ello.

Fuentelsaz y González (2015) analizan el contexto institucional - que, según Kantis et al. (2015), está representada en los diferentes actores que interactúan en los ecosistemas de emprendimiento- y la calidad del emprendimiento como factores que inciden sobre las tasas de fracaso empresarial. En este sentido, el contexto institucional se entiende como todas las reglas de juego que establecen el marco en el que las organizaciones llevan a cabo sus actividades, liderado por instituciones de carácter formal que representan estructuras codificadas y reglas explícitas relacionadas con ámbitos políticos, legales y económicos. Este contexto institucional se hace especialmente importante para las empresas en etapas tempranas, que es cuando requieren un mayor apoyo y oferta de servicios que les facilite el cumplimiento de sus objetivos.

Las instituciones que participan en los ecosistemas de emprendimiento combinan elementos culturales, sociales, políticos y económicos dentro de un contexto 0 territorio para apoyar el desarrollo y crecimiento de nuevas empresas, y alientan a los emprendedores nacientes y a otros actores a asumir los riesgos de iniciar, financiar y ayudar las ideas de emprendimiento (Spigel, 2017). Según lo anterior, un ecosistema de emprendimiento es una comunidad de 
negocios, apoyada por leyes y prácticas, formada por una base de organizaciones e individuos interactuantes que producen y asocian ideas de negocios, habilidades y recursos financieros y no financieros que resultan en empresas dinámicas (Bedoya et al., 2020, p. 97).

Garba y Tsauni (2016) indican que el apoyo e incentivos a emprendimientos no productivos provoca el declive económico y solo los gobiernos y las instituciones pueden generar restricciones para controlar aspectos negativos como la corrupción y políticas laxas que ponen en condición de desventaja a los emprendimientos productivos. Desai et al. (2013) proponen tres supuestos a partir de los cuales se establece y se define la existencia de emprendimientos destructivos; el primero indica que la fuente de talento emprendedor es constante, pero varía en su asignación en búsqueda de maximizar siempre su utilidad, por lo cual la naturaleza de los emprendimientos no es siempre positiva. Un segundo supuesto indica que los emprendedores pueden disminuir sus insumos para la producción actuando como emprendimientos destructivos, malversando las inversiones y destruyendo la posibilidad de generar mayores ingresos futuros. Finalmente, un tercer supuesto determina que los emprendedores son heterogéneos dadas las distintas condiciones iniciales, expectativas y retornos esperados por sus actividades.

Con respecto a las instituciones y los incentivos que tienen los emprendedores para recurrir a ellas, los autores afirman que son indispensables para el desarrollo del talento emprendedor y que sus decisiones se basan en las recompensas que esperan, dadas las limitaciones institucionales que tienen. En este sentido, los emprendedores pueden desarrollarse de forma endógena o exógena a las instituciones, manteniendo siempre el potencial de volverse endógenas si dependen en mayor medida de la ruta ofrecida por las instituciones, con el fin de fomentar el crecimiento económico (Lee et al., 2021). Por lo anterior, la estructura de recompensas para emprendedores determina no solo la asignación o actividad empresarial sino también las futuras estructuras de recompensas.

En cuanto a los antecedentes empíricos, se tiene que:

La literatura institucional resalta tres dimen-
siones políticas que determinan el dinamismo
del emprendimiento: la educación y la cultu-
ra; las acciones de apoyo y la legislación. Las
políticas de educación y cultura se orientan en
instruir y motivar a las personas a emprender.
Las políticas de apoyo consisten en dar sopor-
te a los nuevos negocios, por medio del acce-
so a recursos financieros y no financieros tales
como consultoría técnica, gerencial, crédito
y seguimiento. Mientras que las políticas le-
gislativas se presentan con el surgimiento de
nuevos estatutos, reglamentos y leyes capa-
ces de respaldar y mejorar el entorno empre-
sarial. (Davari y Farokhmanesh, 2017, como se
citó en Osorio et al., 2019, p. 101)

En este sentido, Osorio et al. (2019) indican que el emprendimiento es el puente entre los factores institucionales - en el marco de las políticas de apoyo- y el crecimiento económico; por ende, la calidad institucional es un elemento que debe estar presente; sin embargo, en diversos estudios se ha demostrado que esta calidad institucional es afectada por externalidades negativas como la corrupción y la falta de acceso a servicios públicos, entre otros.

Existen varios estudios empíricos del efecto de la calidad institucional en el emprendimiento. Méndez-Picazo et al. (2012) definen gobernabilidad como el comportamiento y rendimiento del gobierno que hace referencia 
a varios procesos históricos, culturales, sociales y políticos. Utilizando un modelo econométrico de mínimos cuadrados generalizados, concluyen que la gobernabilidad afecta positivamente el emprendimiento, y genera crecimiento económico.

Usando un modelo de regresión logística, Urbano y Álvarez (2014) analizaron el efecto de las dimensiones institucionales en la probabilidad de emprender; específicamente plantean tres tipos de dimensiones institucionales a nivel individual: cultural-cognitiva, normativa y regulativa; encontrando que las dimensiones institucionales aumentan la probabilidad de ser emprendedor. Por otra parte, Samadi (2018), por medio de un análisis empírico, encuentra que existe una relación causal bidireccional entre las instituciones y el proceso emprendedor, dado que cada tópico tiene un rol significativo para generar cambios en el otro.

Osorio et al. (2019) plantean la relación entre la actividad emprendedora y algunas de las conductas públicas, utilizando las variables del acceso y apertura gubernamental, la percepción de la inseguridad y la calidad de los servicios públicos; usando datos de corte transversal y un modelo de probabilidad lineal, encontraron que la calidad de los servicios públicos, el acceso y apertura gubernamental son elementos institucionales significativos al momento de iniciar un negocio en diversas regiones de México, mientras que la percepción de la inseguridad no resulta ser estadísticamente significativa.

Debido a la condición de vulnerabilidad de la población víctima, son frecuentes los emprendimientos por necesidad; por ende, en el presente artículo se tendrán en cuenta factores como capacitación, consultoría y nivel educativo de los emprendedores, que son decisivos para mejorar la competitividad y la sostenibilidad de estos emprendimientos. Estos factores, a la vez, definen la calidad institucional de la región (Mungaray et al., 2016).

\section{Metodología}

En el presente escrito se categoriza a los emprendedores de acuerdo con Brück et al. (2013a), quienes indican que en un escenario de conflicto armado, se adopta una visión más amplia del emprendimiento, que incluye el autoempleo informal y los pequeños agricultores 0 agricultores de subsistencia que ejercen actividades asociadas a su conocimiento ancestral y las actividades tradicionales que realizaban antes de sufrir los hechos victimizantes.

Aquí se presenta un método descriptivo y empírico con datos de corte transversal, recolectados por medio de una encuesta, a 200 personas víctimas del conflicto armado mayores de edad, ubicados en Buga y Tuluá (Valle del Cauca - Colombia); su ubicación y selección se realizó con el apoyo del Observatorio para la Paz del Valle del Cauca ${ }^{7}$, que ya había adelantado actividades de reconstrucción de tejido social y diálogo con la comunidad. El análisis empírico se realizó mediante el uso de Mínimos Cuadrados Ordinarios.

En la sistematización se usó Eviews para el análisis de los datos cuantitativos, para el análisis estadístico descriptivo, para el cruce de variables, para la obtención de las matrices de correlación y la estimación del modelo econométrico. 
Se utilizaron las matrices de correlación para valorar la asociación entre dos variables cuantitativas estudiando el método; la medición de la relación lineal entre dos variables se realiza por medio del cálculo del coeficiente de correlación de Pearson; dicho coeficiente oscila entre $-1 \mathrm{y}+1$. Un valor de -1 indica una relación lineal o línea recta positiva perfecta y una correlación próxima a cero indica que no hay relación lineal entre las dos variables (Gujarati y Porter, 2010), teniendo en cuenta que la correlación no implica causalidad. Se realizó la estimación de un modelo de probabilidad lineal (MPL) utilizando Eviews y se hizo el ajuste al problema de heterocedasticidad usando el mismo software.

De acuerdo con lo planteado por Osorio et al. (2019), para el desarrollo empírico de esta investigación se implementa un modelo lineal de probabilidad (MPL) ${ }^{8}$, empleando el método de Mínimos Cuadrados Ordinarios, y utilizando los datos de corte transversal obtenidos en la encuesta ya mencionada.

Basados en los estudios empíricos citados, se define la variable dependiente como la existencia de actividad emprendedora que sería explicada por un grupo de variables institucionales como el acceso a programas y fuentes de financiación para el emprendimiento y formalización del mismo, conectividad, infraestructura productiva y servicios públicos, a la compensación como víctimas y a los recursos de entidades financieras comerciales; además del conocimiento de la existencia de políticas o normatividad para el fomento del emprendimiento.
En este sentido, el modelo en este caso sería:

$$
\begin{gathered}
\text { EMPR }=\beta_{1}+\beta_{2} \text { Apoyfin }+\beta_{3} \text { Apoynofin } \\
+\beta_{4} \text { Capac }+\beta_{5} \text { Compens }+\beta_{6} \text { Conec } \\
+\beta_{7} \text { Infraest }+\beta_{8} \text { Normformal }+\beta_{9} \text { Prestamos } \\
+\beta_{10} \text { Progapoy }+\varepsilon \text { i }(1)
\end{gathered}
$$

Donde la existencia de actividad emprendedora (EMPR) toma el valor de 1 para las personas que son emprendedoras; apoyo financiero (APOYFIN) toma el valor de 1 cuando ha recibido apoyos financieros por parte de alguna entidad; apoyo no financiero (APOYNOFIN) toma el valor de 1 cuando ha recibido ayuda en especie de cualquier entidad (excluye formación o capacitación); actividades de capacitación (CAPAC) toma el valor de 1 cuando la persona ha recibido algún tipo de capacitación para emprendimiento; compensación o reparación (COM PENS) toma el valor de 1 cuando ha recibido algún tipo de compensación por su carácter de víctima; acceso a conectividad (CONEC) toma el valor de 1 si al menos tiene acceso a conectividad o comunicación personal como teléfono celular o computador; infraestructura (INFRAEST) toma el valor de $1 \mathrm{si}$ al menos tiene acceso a una obra de infraestructura que le permita desarrollar de mejor forma la actividad emprendedora; actividades de normalización o formalización de emprendimientos (NORM FORMAL) toma el valor de 1 cuando ha realizado alguna acción para formalizar el emprendimiento 0 ya está formalizado; acceso a recursos financieros de la banca comercial (PRESTAMOS) toma el valor de 1 cuando ha realizado un préstamo bancario; y, porúltimo, el conocimiento de programas o políticas de apoyo al emprendimiento

8. En este modelo no se cumple que los errores están distribuidos normalmente, pero el no cumplimiento del supuesto de normalidad quizá no sea tan crítico como parece porque sabemos que las estimaciones puntuales de mínimos cuadrados ordinarios (MCO) aún permanecen insesgadas (recuerde que, si el objetivo es la estimación puntual, el supuesto de normalidad resulta innecesario) (Gujarati y Porter, 2010, p. 544). 
(PROGAPOY) toma el valor de 1 cuando las personas indican que conocen los programas o políticas de apoyo al emprendimiento a nivel local.

En cuanto a los efectos esperados, de acuerdo con Urbano y Álvarez (2014), el entorno institucional define, crea y limita las oportunidades empresariales y, por tanto, afecta a las tasas de actividad empresarial; así mismo, las normas y políticas, al igual que la infraestructura productiva, afectan el comportamiento y desarrollo de los emprendimientos.

Por ende, las variables explicatorias definidas afectan de manera positiva la variable dependiente; es decir, aumenta la actividad emprendedora (los signos esperados de todos los coeficientes del modelo son positivos).

\section{Resultados y análisis o discusión}

Descripción de algunas características demográficas y del contexto de la población víctima encuestada

En Colombia el fomento al emprendimiento hace parte de la política pública con normas como la Ley 1014 de 2006 y la Ley 2069 de 2020 de fomento al emprendimiento; en concordancia con esto se han creado ecosistemas con entidades que participan de forma descentralizada en los diferentes departamentos y municipios de la región. De forma particular, para la población estudiada localizada en Buga y Tuluá, varias instituciones tienen presencia en la región con el fin de promover, fomentar y acompañar en algunas fases del proceso el emprendimiento desarrollado en la localidad; teniendo en cuenta esto es preciso conocer cómo es el relacionamiento que ha tenido la población con los diferentes programas, identificando qué tipo de apoyo han recibido, cómo es el reconocimiento de dichasinstitucionesysus programas y el apoyo institucional para garantizar acceso a infraestructura pertinente para la innovación y la tecnología de los emprendimientos.

El análisis descriptivo realizado a la población víctima encuestada que desempeña una actividad emprendedora, incluye a quienes se describen como empresarios activos o que trabajan de manera independiente.

En primer lugar, se puede analizar la actividad emprendedora de la población encuestada, la cual está compuesta por diversas vivencias, orígenes y enfoques que llevan a las personas a poner en práctica sus habilidades emprendedoras. El $36 \%$ de los encuestados declara que, además de ser emprendedor, tiene un rol de empleado; mientras el porcentaje restante indica dedicarse exclusivamente a su emprendimiento, empresa 0 actividad independiente. El análisis de correlación indica que hay un nivel de correlación menor entre algunas variables; por ejemplo, la variable actividad emprendedora presenta una correlación positiva muy débil con la variable acceso a préstamos $(0,13)$, al igual que la variable acceso a conectividad $(0,11)$, lo cual quiere decir que entre mayor sea el número de personas que tengan una actividad emprendedora, también es levemente mayor el número de personas que acceden a préstamos ya algunos servicios relacionados con la conectividad.

Con respecto al nivel de ingresos, las personas encuestadas que tienen en marcha una iniciativa de emprendimiento registran un ingreso promedio de $\$ 554.155$, valor inferior al salario mínimo legal vigente establecido en Colombia de $\$ 828.116$ en 2019 , denotando una evidente precariedad en los ingresos en dicha población. 
Los préstamos son una variable que refleja las posibilidades de acceso a financiación con las que cuenta la población en estudio. De las personas encuestadas, solo el $30 \%$ indica tener acceso a préstamos de diversos orígenes, mientras la mayoría restante no lo hace, limitando y retrasando las posibilidades de crecimiento de sus actividades económicas. La correlación existente entre la actividad emprendedora y los préstamos es muy débil mientras que las personas informan tener préstamos activos con entidades como bancos, cooperativas, fondos financieros y, finalmente, préstamos gota a gota.

Por otra parte, la variable participación en programas de apoyo del Estado es fundamental como medio para la mejorar las condiciones iniciales de los emprendedores de la población víctima, siendo esta una de las formas claras de intervención. Del total de las personas encuestadas que se consideran emprendedores 0 empresarios, el $57 \%$ indicó haber participado en dichos programas estatales de apoyo, aprovechando los beneficios ofrecidos, principalmente en los programas de Familias en acción y los creados por la Unidad para las Víctimas. Es así como los programas de apoyo del Estado no presentan un nivel de correlación significativo con la variable actividad emprendedora $(-0,04)$.

En relación con la variable de acceso a conectividad, es claro que existe una brecha tecnológica en la población en estudio, puesto que solo el $21 \%$ de los encuestados tiene acceso a un equipo de cómputo y el $33 \%$ acceso al servicio de Internet. El acceso a la telefonía celular es el único medio de conectividad al cual acceden la totalidad de los encuestados, pero es innegable que la falta de acceso a las tecnologías de la información y comunicación, sumado a la escasa capacitación en las mismas, genera un rezago competitivo que dificulta el desarrollo y crecimiento de las iniciativas emprendedoras de la población víctima. Con respecto al nivel de correlación, hay una muy baja correlación positiva con la variable préstamos $(0,11)$, ventajas competitivas $(0,14)$, el conocimiento de normas de creación empresarial $(0,18)$ y, finalmente, las capacitaciones tomadas durante los últimos meses $(0,30)$.

De la misma manera, las ventajas competitivas en la zona generan las condiciones básicas necesarias para el surgimiento de las iniciativas empresariales. En cuanto a la percepción de estas variables por parte de la población víctima encuestada, sobresalen especialmente los servicios públicos adecuados para el desarrollo de actividades económicas, vías adecuadas para el transporte de productos, las actividades de capacitación para los emprendimientos en la zona; además, las personas consideran que en la zona se realizan actividades para mejorar los procesos, productos y formas de manejar los negocios.

Las variables ventajas competitivas y condiciones productivas no indican una correlación significativa con la mayoría de las variables en estudio, a excepción de la capacitación, el apoyo no financiero recibido y el acceso a conectividad, con las cuales se evidencia una correlación positiva muy débil.

Así mismo, es importante recordar que la población participante en este estudio ha tenido un desarrollo emprendedor, dentro de un contexto de superación y resiliencia ante las afectaciones del conflicto. En este sentido, es necesario que la población pueda acceder a los servicios de las entidades que se encargan de fomentar y promover el emprendimiento; además, dadas las condiciones de víctimas de la población, se han generado programas para su reparación, lo cual también 
contribuye de alguna manera a esos esfuerzos por superar las diferentes situaciones ocasionadas por el conflicto. En este sentido, en términos de compensación y reparación, solo el $57 \%$ de la población participante en la investigación ha recibido este tipo de beneficios, siendo en su mayoría dinero $(50,9 \%)$, subsidios ( $17 \%$ ) o víveres ( $13 \%)$; en contraste, solo el $4 \%$ ha recibido tierras como compensación. Esta variable tiene una relación positiva, aunque baja $(0,20)$, con respecto a la actividad emprendedora, y al conocimiento de los programas y políticas de apoyo al emprendimiento $(0,25)$; esto evidencia la importancia de aumentar la cobertura y la buena gestión en la distribución de estos recursos en la población que lo requiere.

Por su parte, la relación de los apoyos institucionales de orden monetario o no monetario tiene una relación positiva con la actividad emprendedora. Con respecto a los apoyos financieros y no financieros la mayoría de la población no ha contado con una amplia cobertura por parte de las entidades de fomento del emprendimiento, ya que el $13 \%$ ha recibido apoyo financiero para crear o fortalecer empresa y menos del $50 \%$ ha tenido apoyo no financiero (capacitaciones, dotación de maquinaria y animales o semillas para la producción, asistencia técnica, proyectos en red, entre otros); en el caso específico de las capacitaciones, se evalúa que más del $70 \%$ de la población no ha recibido ningún tipo de capacitación en los últimos 6 meses.

Entre estos tipos de apoyos, los monetarios tienen una relación positiva con la actividad emprendedora $(0,06)$, pero es menor en comparación con los no financieros $(0,22)$, y este a su vez tiene una relación positiva con acceso a préstamos $(0,27)$, desarrollo de capacitaciones $(0,24)$, recepción de compensación $(0,22)$ y el conocimiento de políticas y programas de apoyo al emprendimiento $(0,19)$. Esto demuestra que crear sinergias por parte de las entidades encargadas de fomentar, apoyar y promover el emprendimiento es importante debido a que a medida que las personas empiezan a tener aproximaciones con el ecosistema les permite conocer y acceder a diferentes servicios para fortalecer su actividad emprendedora. Y esta necesidad es aún más latente al identificar que más del $60 \%$ no conoce programas de apoyo de creación y fortalecimiento empresarial; el $82 \%$ desconoce las normas o procesos para crear empresa y un $63 \%$ no reconoce las entidades de apoyo para la creación o fortalecimiento empresarial.

La infraestructura y las relaciones empresariales son igualmente fundamentales para el avance de las empresas, por tanto es necesario contar con vías de acceso y con proveedores de materias primas e insumos para desarrollar la actividad económica; en este orden de ideas, la mayor parte de la población (55 \%) manifiesta que no considera que tenga facilidad para acceder a estas materias primas, insumos o materiales; teniendo en cuenta esto, y la relación positiva $(0,07)$ de esta variable con la actividad emprendedora, es claro que se debe desarrollar, consolidar y mantener el relacionamiento entre productores y proveedores en el departamento y los municipios vecinos para crear redes de distribución que finalmente propendan a mejorar el emprendimiento en el Valle del Cauca.

Las instituciones son básicas para el desarrollo empresarial de la población; a partir de sus programas, políticas y entidades, logran dinamizar u obstaculizar el crecimiento económico de la población, por eso es necesario que se creen programas que contribuyan al crecimiento y madurez de las empresas, de tal forma que puedan impactar positivamente 
la sociedad y aún más cuando se sabe que los recursos invertidos en la población para capacitar o apoyar financieramente tienen un efecto positivo en el emprendimiento.

\section{Análisis empírico}

En la Tabla 1se presentan los resultados de la estimación del modelo, una vez se realizó la corrección de la presencia de heterocedasticidad ${ }^{9}$ propia de los modelos MPL (Gujarati y Porter, 2010, p. 545).

Según la Tabla 1, solo cuatro de los nueve coeficientes de las variables exploratorias son estadísticamente significativos al $5 \%$ y $10 \%$ y presentan los signos esperados (excepto el conocimiento de programas o políticas de emprendimiento). Así, las variables de la ayuda de programas de apoyo financiero, el acceso a capacitación, las acciones para formalizar o normalizar los emprendimientos y el acceso a préstamos de la banca comercial no son estadísticamente significativas para explicar la actividad emprendedora de la población víctima sujeta a estudio ${ }^{10}$.

Por otra parte, en cuanto a las variables significativas en el modelo, se encuentra el acceso a apoyos no financieros (dotación en especie), que incrementan en 16,6 puntos porcentuales la probabilidad de la población de realizar una actividad emprendedora ${ }^{11}$; esto es relevante, dado que para esta población los apoyos técnicos y de insumos son un mayor incentivo para emprender que, como ya se observó, los apoyos financieros.
Tabla 1

Modelo MPL.

\begin{tabular}{|c|c|c|}
\hline Variables & Coeficiente & Valor t \\
\hline INTERCEPTO & 0,16837 & $* * 1,7911$ \\
\hline APOYFIN & $-0,00232$ & $-0,0205$ \\
\hline APOYNOFIN & 0,16601 & $* 2,1367$ \\
\hline CAPAC & 0,07925 & 0,8500 \\
\hline COMPENS & 0,18106 & $* 2,5791$ \\
\hline CONEC & 0,04521 & $* * 1,9758$ \\
\hline INFRAEST & 0,08754 & $* * 1,9404$ \\
\hline NORMFORMAL & 0,01539 & 0,19592 \\
\hline PRESTAMOS & 0,05358 & 0,61339 \\
\hline PROGAPOY & $-0,12847$ & $* * 1,8580$ \\
\hline $\mathrm{R}^{2}$ & & 0,2726 \\
\hline Prob F statics & & 0,0090 \\
\hline \multicolumn{3}{|c|}{$\begin{array}{l}\text { - Los parámetros *significativos al } 5 \% \text { y **significativos al } 10 \% \text {. } \\
\text { - El R2 calculado de manera convencional tiene un valor limitado } \\
\text { en los modelos de respuesta dicótoma. En la mayoría de las apli- } \\
\text { caciones prácticas, R2 se encuentra entre } 0,2 \text { y } 0,6 \text { (Gujarati y } \\
\text { Porter, 2010, p. 546). }\end{array}$} \\
\hline
\end{tabular}

Fuente: elaboración propia, usando Eviews.

Cuando las personas han recibido compensación o reparación, la actividad emprendedora se incrementa en 18,1 puntos porcentuales; es decir, cuando las personas han recibido su reconocimiento económico como víctimas, están más dispuestas a llevar a cabo un emprendimiento. Así mismo, si las personas han tenido acceso a un medio de conectividad, como un teléfono celular, computador o Internet, incrementan en 4,5 puntos porcentuales la probabilidad de realizar una actividad emprendedora.

9. Una vez ajustados los problemas existentes de heterocedasticidad, se pueden revisar los resultados de los parámetros en este modelo (Rosales et al., 2010).

10. Los resultados registrados deben ser tomados como la representación de una tendencia, porque son dados para una muestra pequeña; no se puede realizar inferencia estadística ni son extrapolables a otros grupos de población.

11. Cuando se tiene variables independientes binarias el coeficiente que se obtiene mide la diferencia que se predice para la probabilidad en relación con el grupo base (Wooldridge, 2009). 
Con referencia a la existencia de infraestructura productiva en la región, como vías de transporte adecuadas, dotación de servicios públicos, entre otros, el resultado de la regresión indica que la posibilidad de acceso a esta aumenta en 8,7 puntos porcentuales la actividad emprendedora de la población analizada. Es claro el impacto que genera sobre la actividad emprendedora la existencia de una infraestructura óptima, de acuerdo con Martínez (2018), para quien era importante esta infraestructura, esencialmente en el sector de las TIC.

Por otro lado, contrario a lo esperado, el conocimiento de programas o políticas de apoyo al emprendimiento por parte de la población reduce en 12,9 puntos porcentuales la posibilidad de llevar a cabo una actividad emprendedora; por lo que se podría inferir que estas políticas o programas de apoyo inducen a la destrucción del emprendimiento; es decir, que el emprendimiento productivo puede llegar a ser reemplazado por un emprendimiento improductivo o destructivo. A esto se suma el hecho de que las variables de apoyo financiero, el acceso a capacitación y las acciones para formalizar o normalizar los emprendimientos, que hacen parte de los programas de fomento para el emprendimiento de entidades públicas y privadas, no son significativas para la actividad emprendedora de la población analizada.

\section{Conclusiones y recomendaciones}

Si bien puede indicarse que el emprendimiento depende, en su mayoría, de la intención propia del emprendedor y su capacidad de resiliencia y con mayor razón en la población objeto de análisis, también es necesario que exista un entorno institucional que permita dar soporte para la consolidación exitosa de dichos emprendimientos.
En este sentido,

Las instituciones pueden considerarse como las reglas del juego que establecen el marco en el que las organizaciones llevan a cabo su actividad (North, 1990). Si estas reglas del juego están claras, la actividad emprendedora se desarrollará en condiciones más favorables, con el consiguiente efecto positivo en la misma. Por el contrario, si el marco institucional no es el adecuado, los mercados serán más opacos y menos competitivos, lo que puede desincentivar la apertura de nuevos negocios. (Fuentelsaz y González, 2015, p. 68)

En cuanto al análisis descriptivo de la actividad emprendedora en la población víctima estudiada, más de la mitad de los encuestados se dedican de lleno a su actividad emprendedora, pero generan ingresos en promedio muy por debajo del salario mínimo legal vigente.

El acceso a préstamos es una variable preocupante, pues solo una tercera parte de los emprendedores encuestados indican tener acceso a financiación formal con entidades como bancos y cooperativas. Por otra parte, la participación en programas del Estado demuestra un mayor nivel de aceptación pues seis de cada diez personas encuestadas indican tener acceso a dichos programas como, por ejemplo, Familias en acción.

La brecha tecnológica es una realidad en esta población, pues solo un cuarto de la población tenía acceso a equipos de cómputo y cuatro de cada diez personas tenían servicio de Internet, situación que contrasta con la percepción de las ventajas competitivas en la zona, pues gran parte de los encuestados considera que los servicios públicos son adecuados para su actividad, piensa que las vías son buenas para transportar sus productos e indica estar conforme con las actividades de capacitación que se dictan en la zona. 
Con respecto al nivel de correlación de la actividad emprendedora con las demás variables, presenta una correlación positiva baja o débil con variables como el acceso a préstamos y servicios de conectividad, mientras que variables como la participación en programas de apoyo del Estado y ventajas competitivas en la zona no evidencian una correlación significativa.

Así mismo, a pesar de que las instituciones son importantes para el desarrollo empresarial de la población, a partir de sus programas, políticas y entidades, logran dinamizar u obstaculizar el desarrollo económico; la mayor parte la población encuestada no percibía facilidad para acceder a materias primas, insumos ni materiales, no habían recibido apoyo financiero, ni conocían sobre los programas, las entidades o las normas para crear empresa; esto se reafirma con la débil correlación de estas variables con la actividad emprendedora, sugiriendo que las instituciones tiene un menor relacionamiento de lo esperado con la población víctima, en la promoción o fomento del emprendimiento.

El ejercicio empírico coincide con lo encontrado en el análisis descriptivo, dado que si bien es cierto que en el país existen múltiples programas basados en apoyos financieros y no financieros, los resultados del modelo indican que, para la población seleccionada, son de mayor relevancia los últimos, al igual que la existencia de infraestructura productiva y el acceso a la conectividad en la región y la compensación o reparación como víctimas para promover su actividad emprendedora. Por otra parte, es necesario revisar el diseño y la forma de divulgación de los programas de fomento al emprendimiento ya existentes, dado que, como puede observarse, contrario a su objetivo, el conocimiento de la existencia de estos programas afecta de manera negativa el emprendimiento.

En este sentido, a pesar de la creación de la nueva política de emprendimiento registrada en el CONPES 4011 (Departamento Nacional de Planeación, 2020), quetiene como objetivo "generar condiciones habilitantes en el ecosistema emprendedor para la creación, sostenibilidad y crecimiento de emprendimientos que contribuyan a la generación de ingresos, riqueza y aumentos en la productividad e internacionalización empresarial" (p. 3), que si bien contempla la necesidad de realizar un enfoque diferencial en la población vulnerable, entre ellas las víctimas, aún no es claro el proceso o la ruta diferencial para esta población sujeto de esta investigación.

Es claro que, de acuerdo con los resultados expuestos, se rechaza la hipótesis de investigación planteada, para esta población en particular. En este sentido, resulta pertinente que futuras investigaciones cuestionen el sentido de causalidad entre las variables institucionales y de emprendimiento, ante diversos actores y contextos regionales y nacionales, dado que es necesario evidenciar el papel de las instituciones de apoyo a las empresas en el entorno débil que ha sido generado por el conflicto (Khattak et al., 2021).

Puesto que, como indicó Acs (2006), cualquier estudio que analice el fenómeno del emprendimiento no debe analizar las nuevas empresas de forma agregada, por el contrario, debe tener en cuenta las diferencias según el tipo de emprendimiento, el emprendedor y su entorno, ya que su efecto sobre la economía puede variar de forma significativa. 


\section{Referencias}

Acs, Z. (2006). How is Entrepreneurship Good for Economic Growth?, Innovations: technology, Governance and Globalization, 1(1), 97-107. https:// doi.org/ 10.1162/ itgg.2006.1.1.97

Aidis, R., Estrin, S. y Mickiewicz, T. (2008). Institutions and entrepreneurship development in Russia: A comparative perspective. Journal of Business Venturing, 23(6), 656-672. https:// doi.org/ 10.1016/ j.jbusvent.2008.01.005

Almodóvar, M. (2018). Tipo de emprendimiento y fase de desarrollo como factores clave para el resultado de la actividad emprendedora. Cuadernos de Relaciones Laborales, 36(2), 225-243.

Bedoya C., Castro, M. Y. y Hoyos, A. (2020). El emprendimiento rural en la construcción de paz: análisis de la (des)articulación en el Valle del Cauca, Colombia. Opera, 27, 91117. https:// papers.ssrn.com/sol3/ papers. cfm?abstract_id $=3620483$

Brainard, L. y Chollet, D. (eds.) (2007). Too poor for peace?: global poverty, conflict, and security in the 21st century. Brookings Institution Press.

Brück, T., Naudé, W. y Verwimp, P. (2013a). Entrepreneurship and violent conflict in developing countries. WIDER Working Paper, No. 28. https:// www.wider.unu.edu/publication/ entrepreneurship-and-violent-conflict-developing-countries

Brück, T., Naudé, W. y Verwimp, P. (2013b). Business underfire: Entrepreneurship and violent conflict in developing countries. Journal of Conflict Resolution, 57(1), 3-19. https:// doi. org/ 10.1177/ 0022002712464846

Cortés-Sánchez, J. D. (2018). Institutional strength, peacebuilding, and productive entrepreneurship-exploratory analysis in Colombia. Verslas: teorija ir praktika, 19(1), 88-102.
Coyne, C. J., Sobel, R. S. y Dove, J. A. (2010). The Non-Productive Entrepreneurial Process. Rev. Austrian Economics, 23, 333-346. DOI: $10.1007 /$ s $11138-010-0124-2$

Departamento Nacional de Planeación (DNP) (2020). Política Nacional de Emprendimiento, Documento CONPES 4011, 30 de noviembre de 2020. https:// colaboracion.dnp.gov. co/ CDT/ Conpes/ Econ\%C3\%B3micos/ 4011. pdf

Desai, S., Acs, Z. J. y Weitzel, U. (2013). A model of destructive entrepreneurship: Insight for conflict and postconflict recovery. Journal of Conflict Resolution, 57(1), 20-40.

Dzafic, Z. y Babajic, A. (2016). The role of the government in entrepreneurship development: Evidence from Bosnia and Herzegovina. Economic Review: Journal of Economics and Business, 14(1), 68-79.

Fuentelsaz, L. y González, C. (2015). El fracaso emprendedor a través de las instituciones y la calidad del emprendimiento. Universia Business Review, 47, 64-81.

Garba, A. S. y Tsauni, A. M. (2016). An Analysis of Productive and Destructive Entrepreneurship: A Survey of Literature. Journal of Social Sciences and Public Policy, 8(1), 32-46.

García, H. (2016). Innovación y emprendimiento en América Latina: desafíos y oportunidades de la región para sumarse a la sociedad del conocimiento: El caso colombiano. En: D. Gregosz (ed.), La fuerza de la innovación y el emprendimiento, (pp. 99-116). https:// bivica.org/ files/innovacion-emprendimiento. pdf\#page $=96$

Gujarati, D. y Porter, D. C. (2010). Econometría básica. McGraw-Hill. 
Joseph, J., Daou, A., Ali, Z., Reyes, G. (2018). Entrepreneurship in conflict zones: Exploring transformative subsistence entrepreneurship. British Academy of Management. https:// bit.ly/ 3r3CNBd

Kantis, H., Federico, J. y Magendzo, A. (2015). Condiciones sistémicas e institucionalidad para el emprendimiento y la innovación: Hacia una agenda de integración de los ecosistemas en los países de la Alianza del Pacífico. Banco Interamericano de Desarrollo.

Khattak, M. N., Muhammad, N. y Robinson, D. (2021). Understanding the interplay between support agencies and small and medium-sized enterprises in a conflict environment from an institutional theory perspective. Asia-Pacific Journal of Business Administration, 13(2), 256-271. https://doi. org/ 10.1108/APJBA-03-2020-0097

Lee, C. K., Wiklund, J., Amezcua, A., Bae, T. J. y Palubinskas, A. (2021). Business failure and institutions in entrepreneurship: a systematic review and research agenda. Small Business Economics, 57(2), 1-27.

LêKhang, T. y Thành, N. C. (2018). Economic growth, entrepreneurship, and institutions: evidence in emerging countries. In The 5th IBSM international conference on business, management and accounting, 19-21 de abril, Hanoi University of Industry, Vietnam. http:// caal-inteduorg.com/ proceedings/ ibsm5/ES6-71.pdf

Martínez, L. F. (2018). Política pública de emprendimiento en Colombia 2006-2016: un análisis cognitivo desde los instrumentos de planificación (Disertación doctoral), Universidad Externado de Colombia.
Méndez-Picazo, M., Galindo-Martín, M. y Ribeiro-Soriano, D. (2012). Governance, Entrepreneurship and Economic Growth, Journal of Entrepreneurship and Regional Development, 24(10), 865-877. https:// doi.org/ 10.1 $080 / 08985626.2012 .742323$

Mungaray, A., Aguilar, J. G. y Osorio, G. (2016). Rethinking the purpose of micro-enterprises in developing countries: Evidence for Mexico. Journal of Developmental Entrepreneurship, 21(3), 1-16. https://doi. org/ 10.1142/ S1084946716500199

Naudé, W. (2009). Entrepreneurship, Post-Conflict. En T. Addison y T. Brück (eds.), Making Peace Work. The Challenges of Social and Economic Reconstruction (pp. 251-263). Palgrave Macmillan. doi: https://doi. org/ 10.1057/ 9780230595194_11

Osorio, G., Saavedra, R. y Martínez, G. (2019). El impacto institucional en el emprendimiento de México. Revista de Estudios Empresariales, Segunda época(1), 98-115.

Rosales, R., Perdomo, J. A., Morales, C. A. y Urrego, J. A. (2010). Fundamentos de Econometría Intermedia: Teoría y Aplicaciones. Universidad de los Andes.

Samadi, A. H. (2018). Institutions and Entrepreneurship in MENA Countries. En: N. Faghih y M. R. Zali (eds.), Entrepreneurship Ecosystem in the Middle East and North Africa (MENA) (pp. 53-93). Springer. https://doi. org/ 10.1007/978-3-319-75913-5_3

Sanders, M. y Weitzel, U. (2013). Misallocation of entrepreneurial talent in postconflict environments. Journal of Conflict Resolution, 57(1), 41-64. https://doi. org/ $10.1177 / 0022002712464852$ 
Sanguinetti, P., Brassiolo, P., Arreaza, A., Berniell, L., Álvarez, F., Ortega, D. y Kamiya, M. (2013). RED 2013: Emprendimientos en América Latina. Desde la subsistencia hacia la transformación productiva (Reporte de Economía y Desarrollo (RED)). CAF. http:// scioteca.caf. com/ handle/ 123456789/ 168

Sauka, A. (2008). Productive, Unproductive, Destructive Entrepreneurship: A Theoretical and Empirical Exploration. William Davidson Institute. Working Paper 917.

Sobel, R. S. (2008). Testing Baumol: Institutional quality and the productivity of entrepreneurship. Journal of Business Venturing, 23(6), 641-655. https:// doi.org/ 10.1016/j.jbusvent.2008.01.004

Spigel, B. (2017). The relational organization of entrepreneurial ecosystems. Entrepreneurship theory and practice, 41(1), 49-72. https:// doi.org/ 10.1111/ etap.12167

Stam, E. (2009). Entrepreneurship, evolution and geography. Papers on Economics and Evolution (0907). Max Planck Institute of Economics, Jena.

Urbano, D. y Álvarez, C. (2014). Institutional dimensions and entrepreneurial activity: an international study. Small Business Economics, 42(4), 703-716.

Urbano, D. y Aparicio, S. (2016). Entrepreneurship capital types and economic growth: International evidence. Technological Forecasting and Social Change, 102, 34-44.

Vorley, T. y Williams, N. (2017). Fostering entrepreneurship and economic growth: Pathways to economic resilience in Kosovo. World Review of Entrepreneurship, Management and Sustainable Development, 13(2-3), 159-177.

Wooldridge, J. (2009). Introductory Econometrics: A modern approach. Cengage Learning.

Zietarski, J. (2018). Legal destructive entrepreneurship in the modern economy. Ekonomia $i$ Prawo. Economics and Law, 17(3), 339-350. https:// doi.org/ 10.12775/ eip.2018.025 